\title{
Two-Dimensional Wigner Crystal on Helium Films: An Indication of Quantum Melting
}

\author{
J. A. R. da Cunha* and Ladir Cândido ${ }^{\dagger}$ \\ $\dagger$ Instituto de Física, Universidade Federal de Goiás Campus Samambaia, 74001-970, Goiânia GO, Brazil and \\ * Instituto de Física, Universidade de Brasília, 70919-970, Brasília, DF, Brazil
}

Received on 7 September, 2005

\begin{abstract}
Using molecular dynamics simulation (MD) we have investigated the melting of the two-dimensional Wigner crystal on $240 \AA-500 \AA$ liquid helium films supported by substrates of dielectric constants $\varepsilon_{s}=2.2-7.3$. Our results show good agreement with available theoretical and experimental results for densities below $1.0 \times 10^{10} \mathrm{~cm}^{-2}$. For higher densities, we notice some disagreements suggesting that quantum effects are important in this regime of densities.
\end{abstract}

Keywords: Wigner crystal; Molecular dynamics; Melting temperature

\section{INTRODUCTION}

It is well established that electrons on helium surface are an almost ideal two dimensional electron system. They form the cleanest example of a two dimensional electron gas that, at sufficiently low densities and temperatures (where the potential dominates), will form a two dimensional electron crystal called Wigner crystal[1]. In the bulk this system undergoes a solid-liquid phase transition at a temperature $T_{m}=$ $2 e^{2}(n \pi)^{1 / 2} /\left(\varepsilon_{H e}+1\right) k_{B} \Gamma_{m}$, which is much higher than the Fermi temperature $T_{F}=\pi n \hbar^{2} / k_{B} m$. Here $\varepsilon_{H e}$ is the dieletric constant of helium, $m$ the electron mass and $\Gamma_{m}$ is the plasma parameter at the melting temperature. Therefore, such electrons obey the classical Boltzmann statistics and their phase diagram can be calculated using classical approach.

Lindeman's melting criterion[2] tells us that the thermodynamic state of a classical coulomb system is determined by the quantity $\Gamma=\langle U\rangle /\langle K\rangle$ which is a measure of the ratio of the mean coulomb potential energy to mean kinetic energy. Thus, for $\Gamma \lesssim 1$ the kinetic energy predominates and the system behaves like an electron gas. At intermediate densities $1 \lesssim \Gamma \lesssim 100$, the electron motion becomes highly correlated or liquidlike. At high densities $\Gamma \gtrsim 100$ the coulomb potential energy predominates and the electrons are expected to undergo a phase-transition to form a periodic crystalline array. Experimentally the liquid-to-solid transition in the bulk takes place for a value of the coupling constant[3] $\Gamma_{m}=137 \pm 15$ and computer simulations by Kalia et al.[4] indicate a firstorder melting $\Gamma_{m}=118-130$ in good agreement with experiment.

Superficial electrons on helium films also form a very interesting system to study the many-body properties of $2 \mathrm{D}$ screened systems. In this case the screening is provided by the image charges in the substrate beneath the film. The screening effect can drastically change the electron-electron interacting potential, going from $1 / r$ to $1 / r^{3}$, through varying external parameters such as film thickness and dielectric constant of the substrate. Peeters [5], using a phenomenological approach got a reduction in the phase diagram of this electron system in comparison to the bulk case. Saitoh[6] using an analytical approximation for the angular frequency of the transverse phonon combined with the Kosterlitz-Thoules melting criterion, got a melting transition in this system in agreement with Jiang et al. experiments[7]. Cândido et al.[8] using computer simulation, studied the thermodynamical, structural and dynamical properties of this two-dimensional electron system. Experimentally, the melting temperature of the Wigner crystal on thin helium films adsorbed on dielectric substrates was measured by Jiang et al. [7] through the electron mobility and by Mistura et al.[9] using microwave cavity technique.

In this paper, we will get back to the discussion pointed out by the authors in a previous work [10] about the discrepancies between simulational and experimental results (at electron densities higher than $1.3 \times 10^{10} \mathrm{~cm}^{-2}$ ) that might be related to the importance of quantum effects at such regime. The obtained results are directly compared with available experimental data by Mistura et al.[9] and Jiang et al. [7], and theoretical results by Peeters[5] and Saitoh[6].

\section{SCREENING COULOMB POTENTIAL}

We consider a two-dimensional system of electrons on a helium film of thickness $d$ adsorbed on a substrate of dielectric constante $\varepsilon_{S}$, interacting through a screening Coulomb potential[11]. The electron system is immersed in a rigid, uniform, positive charge background to make a neutral charged system. The interaction potential between two electrons on the helium film with thickness $d$ (the distance between the electron layer and the substrate), wich incorporates the remarkable influence of the different film thickness and substrates can be obtained through the solution of Poisson equation with appropriate boundary condition, is given by

$$
V(r)=2 e^{2}\left[\frac{1}{\left(1-\varepsilon_{H e}\right) r}-\frac{\varepsilon_{H e}}{\left(1+\varepsilon_{H e}\right)} \sum_{n=1}^{\infty} \frac{\delta^{2}\left(\frac{1-\varepsilon_{H e}}{1+\varepsilon_{H e}}\right)}{\sqrt{r^{2}+(2 n d)^{2}}}\right],
$$


where $\delta=\left(\varepsilon_{s}-\varepsilon_{H e}\right) /\left(\varepsilon_{s}+\varepsilon_{H e}\right)$, with $n$, $\varepsilon_{s}$ and $\varepsilon_{H e}$ being the electron density, the dielectric constants of the substrate and helium film respectively. If we approximate the dielectric constant of the helium film by $1\left(\varepsilon_{H e}=1.057\right)$, the series in the second term of Eq. 1 converges rapidly, and we can rewrite the interaction potential as

$$
V(r)=e^{2}\left[\frac{1}{r}-\frac{\delta}{\sqrt{r^{2}+(2 d)^{2}}}\right] .
$$

The second term of Eq. 2 gives the screening due to the constant of substrate and film thickness. For small interparticle distances $r_{0} \ll d$, where $r_{0}=(\pi n)^{-1 / 2}$ is the mean distances between electrons, the screening is negligible and one has a Coulombic potential

$$
V(r)=\frac{e^{2}}{r}
$$

If instead the electrons are far apart, $r_{0} \gg d$, and we have

$$
V(r)=\frac{e^{2}(1-\delta)}{r}+\frac{2 \delta e^{2} d^{2}}{r^{3}}+\frac{6 \delta e^{2} d^{4}}{r^{5}}+\cdots .
$$

Note that for a metallic substrate, $\varepsilon_{s}=\infty$ where $\delta=1$, the interaction is between dipoles of strenght $p=2 e d$ composed of electrons and their images in the substrate

$$
V(r)=\frac{2 e^{2} d^{2}}{r^{3}}
$$

\section{MOLECULAR DYNAMICS PROCEDURE}

In this work most of the molecular dynamics calculations were performed on a system of 100 electrons with a few runs of 484 and 784 electrons to study size effects. The finite size effect is investigated by changing the system size and the thermodynamical behavior in an infinite system is derived from its extrapolation. The initial positions of the electrons are in a triangular lattice which is accommodated in a rectangular box with periodic boundary condictions to eliminate the surface effects. Because of the long-range nature of the electron-electron and electron-background interacting potential we employ the Ewald summation which splits the potential into a long-range and a short-range part. The long-range part is handled in $k$ space, while the short range part in the real space. We have used the fifth-order predictor-corrector algorithm to integrate Newton's equation of motion with the MD time step varying from $10^{-12}$ to $10^{-15} \mathrm{sec}$, since it has some scale dependence on the electron densities. The optimum time step leads to a conservation of the total energy of 1 part in $10^{4}$ after several thousands time step runs. The time averages of the physical quantities were obtained over 120000 time steps after the system has reached the equilibrium. The transition temperature is obtained performing a simulation in two steps: (i) a sequence of runs in cascade coming down from high to low temperatures at time step fixed and stoping when the system freezes at the temperature $T_{\text {freezes }}$, (ii)

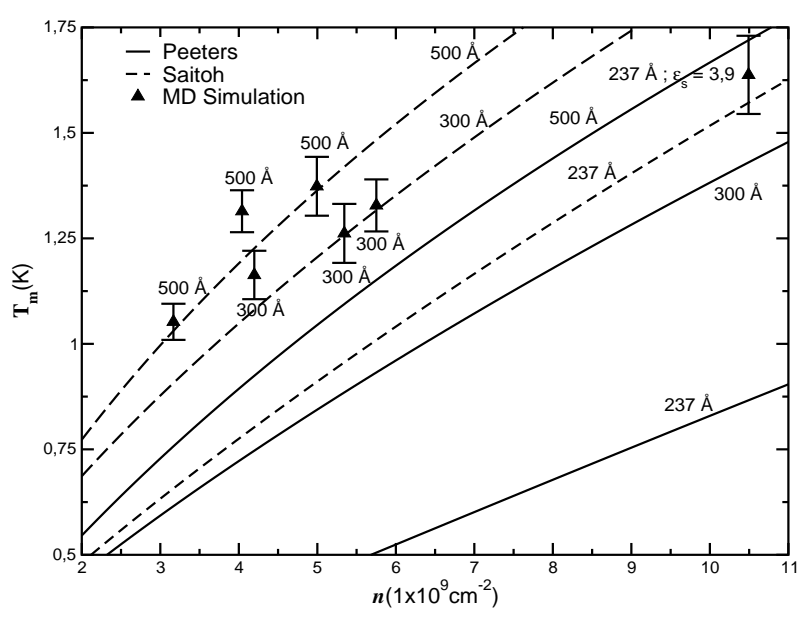

FIG. 1: The melting temperature as a function of the electron density for dielectric constants of 2.2 and 3.9.

a sequence of runs starting at very low temperature (tringular lattice) with the time step fixed stopping when the system melts at the temperature $T_{\text {melting }}$. The transition temperature is given by $T_{\text {freeze }}<T_{m}<T_{\text {melting. }}$. In the discussion of the results below we call $T_{m}$, the transition temperature, as the melting temperature.

\section{RESULTS AND DISCUSSIONS}

Figures 1-3 show the melting temperature as a function of the electron density at several film thicknesses and dielectric constants of the substrates. Because it is quite difficult to plot all the available data (simulational, theoretical and experimental) in one single graphic, we will discuss the results using three separate plots. Fig. 1 shows our simulation results and Saitoh's[8] and Peeters's[7] theoretical results. One can notice an agreement with Saitoh's and a disagreement with Peeters's. This can be justified as being a possible double counting in the calculation of $T_{m}$ by Peeters, as already pointed by Saitoh. In Fig. 2 we campare our results to Jiang's et al.[10] experiment. To emphasize the differences between the three types of results we add to this plot the theoretical results by Saitoh. There is a good agreement between the three types of results, except at the density $1.3 \times 10^{10} \mathrm{~cm}^{-2}$. In Fig. 3 we give a comparison with the experiment by Mistura et al. . For densities below $5 \times 10^{9} \mathrm{~cm}^{-2}$ we obtain a good concordance. Again, for densities higher than $1 \times 10^{10} \mathrm{~cm}^{-2}$ the results do not agree, even though the differences in $T_{m}$ at such densities are almost within the uncertainty in the experimental measuremment. We also show in this figure the good concordance with Saitoh's theoretical results for all densities studied. A possible explanation for such discrepancies could be that quantum effects are important at such densities, which corroborates the measuremments taken by Günzler et al.[12] using microwave cavity technique at $T=1.2 \mathrm{~K}$. These measurements show an abrupt increase of the electron mobility at electron densities near $10^{11} \mathrm{~cm}^{-2}$, which is suggested as quantum melting of the Wigner solid. In addition to this point, 


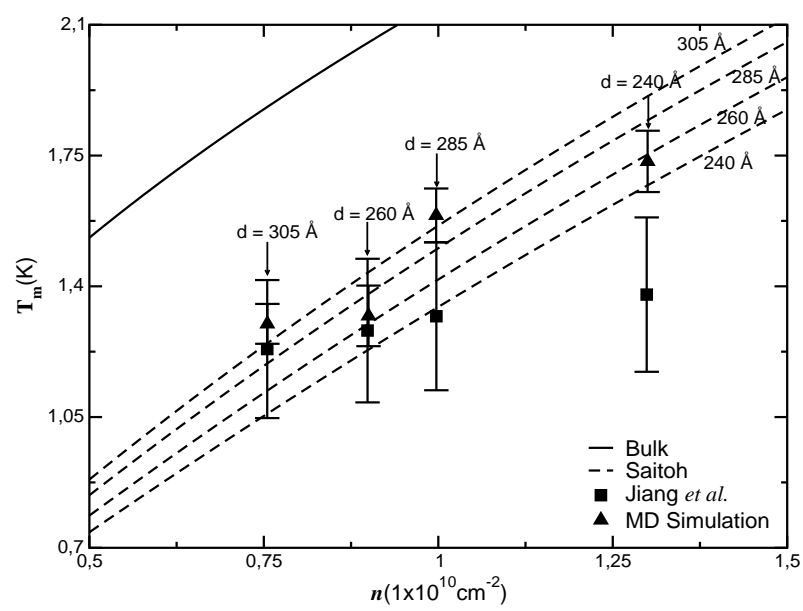

FIG. 2: The melting temperature as a function of the electron density for different film thicknesses and dielectric constant of 7.3. The solid line is the bulk limit.

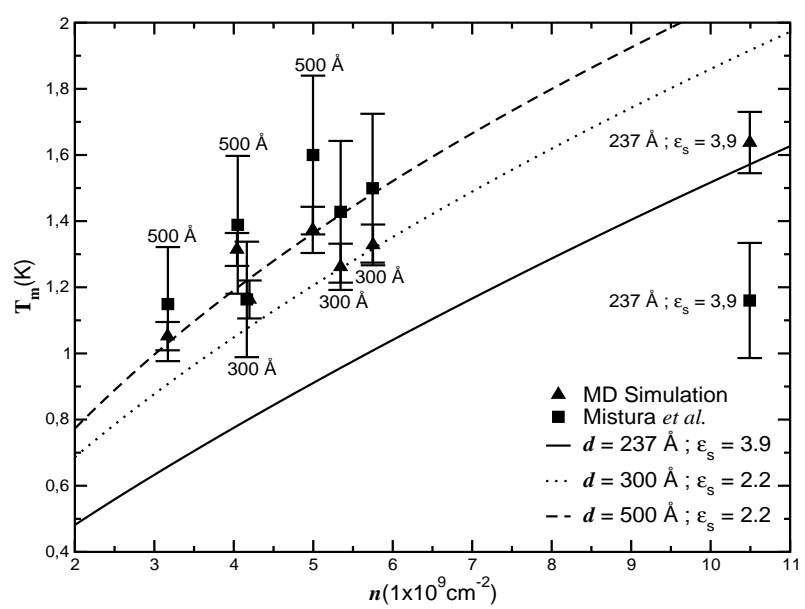

FIG. 3: The melting temperature versus the electron density for diferent film thicknesses and two dielectric constants (2.2 and 3.9). we note that the change in entropy on melting is found to be decreasing as one increases (decreases) the density or the dielectric constant of the substrate (the film thichness). It might imply that transition becomes continuous at higher densities.

\section{CONCLUSIONS}

We have shown in this work that the MD is able to reproduce the experimental measurements of the melting temperature in the two-dimensional electrons on thin liquid $\mathrm{He}$ films for densities below $1.0 \times 10^{10} \mathrm{~cm}^{-2}$. For higher densities we notice some discrepancies with the available experimental measuremments, suggesting that at such densities quantum effects are important and our results using classical molecular dynamics method might be beyond its applicability.

\section{Acknowledgments}

This research was partially sponsored by Conselho Nacional de Desenvolvimento Científico e Tecnológico (CNPq) and Fundação Nacional de Apoio a Pesquisa (FUNAPEUFG). J.A.R. da Cunha is supported by Conselho Nacional de Desenvolvimento Científico e Tecnológico (CNPq).
[1] E. Wigner, Phys. Rev. 46, 1002 (1934).

[2] P. M. Platzman and H. Fukuyama, Phys. Rev. B. 10, 3150 (1974).

[3] C. C. Grimes and G. Adams, Phys. Rev. Lett. 42, 795 (1979).

[4] R. K. Kalia and P. Vashishta, Phys. Rev. B. 23, 4794 (1981).

[5] F. M. Peeters, Phys. Rev. B. 30, 159 (1984). F. M. Peeters and P. M Platzman, Phys. Rev. Lett. 50, 2021 (1983).

[6] M. Saitoh, Phys. Rev. B. 40, 810 (1989).

[7] H.-W. Jiang and A. J. Dahm, Phys. Rev. Lett. 62, 1396 (1989); Surf. Sci. 1961 (1988), ibid. 229, 352 (1990).

[8] L. Cândido, J. P. Rino, and N. Studart, Phys. Rev. B. 54, 7046
(1996).

[9] G. Mistura, T. Günzler, S. Neser, and P. Leiderer, Phys. Rev. B. 56, 8360 (1989).

[10] J. A. R. da Cunha and L. Cândido, Phys. Rev. B. 71, 073403:14(2005).

[11] W. R. Smythe, Static and Dynamic Electricity (McGraw-Hill, New York, 1950), Pag. 182.

[12] T. Günzler, B. Bitnar, G. Mistura, and S. Neser, P. Leiderer Surf. Sci. 361/362, 831 (1996). 